\title{
Development of Walking Assistive Cane for Obstacle Detection and Location Recognition for Visually Impaired People
}

\author{
Chang-Min Yang, ${ }_{1}^{1}$ Ji-Yong Jung, ${ }^{2}$ and Jung-Ja Kim ${ }^{2,3 *}$ \\ ${ }^{1}$ Department of Healthcare Engineering, Jeonbuk National University, \\ 567 Baekje-daero, Deokjin-gu, Jeonju-si, Jeollabuk-do 54896, Republic of Korea \\ ${ }^{2}$ Divison of Biomedical Engineering, Jeonbuk National University, \\ 567 Baekje-daero, Deokjin-gu, Jeonju-si, Jeollabuk-do 54896, Republic of Korea \\ ${ }^{3}$ Research Center of Healthcare \& Welfare Instrument for the Aged, Jeonbuk National University, \\ 567 Baekje-daero, Deokjin-gu, Jeonju-si, Jeollabuk-do 54896, Republic of Korea
}

(Received July 21, 2021; accepted October 20, 2021)

Keywords: visually impaired people, walking assistive cane, obstacle detection, ultrasonic sensor, RFID

Visually impaired people generally use a white cane for safe walking owing to its advantages of familiarity, reliability, low cost, and usability, but it is difficult to detect low obstacles with a cane. Although electronic walking assistive canes equipped with various sensors are being developed to solve several problems, these devices cannot be used by visually impaired people owing to their very high prices and insufficient reliability. We have developed a new walking assistive cane for visually impaired people that uses three ultrasonic sensors, a vibration motor, a radio frequency identification (RFID) reader and tags, a speaker, a cadmium sulfide photoresistor, and an LED. An experimental environment with cylindrical obstacles and RFID was designed to evaluate its effectiveness. Ten participants with normal vision were recruited, and they walked blindfold through the experimental environment three times using a normal white cane and three times using the developed cane. In the experiment, walking with the developed cane showed higher obstacle detection performance than walking with a normal white cane. However, the walking speed of the user was lower using the developed cane. According to the results of a questionnaire on the satisfaction of walking with the developed cane, recognition of the current location became easier with the use of RFID but the detection range of RFID should be further increased. The cane developed in this study can be manufactured inexpensively, and the effect of the weight of the cane on the user is reduced by attaching a wheel to its tip to allow it to be pushed forward rather than swung from side to side. This is a basic study on improving the satisfaction of spatial perception ability when transmitting spatial information to visually impaired people using RFID, and its results can be used to develop electronic travel aids in the future.

\section{Introduction}

There are about 253 million visually impaired people in the world (blind: 36 million, low vision: 217 million). ${ }^{(1)}$ They often use a walking assistive device for independent walking, which

${ }^{*}$ Corresponding author: e-mail: jungjakim@jbnu.ac.kr https://doi.org/10.18494/SAM.2021.3452 
is important because it provides confidence to individuals. ${ }^{(2)} \mathrm{A}$ white cane is the most common walking assistive device owing to its advantages of accessibility, safety, and usability. In addition, it can provide the user with tactile information such as the position and size of detected obstacles on the ground. However, until now, visually impaired people have had difficulties in detecting low-lying obstacles and in determining the direction to their destination, which may have resulted in increased accidents or injuries. ${ }^{(3)}$ To solve these problems, many electronic walking assistive devices with various sensors have recently been developed. Shoval et al. ${ }^{(4)}$ developed a guide cane using 10 ultrasonic sensors and a servo motor. It was controlled with the servo motor using a joystick since the weight of the cane was increased by the sensors for detecting obstacles. Wahab et al. ${ }^{(5)}$ manufactured a smart cane with a speaker and vibrating gloves for users with poor hearing. However, since it was developed as a prototype and has high power consumption, it requires more battery packs than a guide cane. Hoyle and Waters ${ }^{(6)}$ developed the ultracane, which uses two ultrasonic sensors and is the only commercially available electronic travel aid. Obstacles higher than the ground and waist can be detected using the ultrasonic sensors, but the price of the ultracane is too high for most visually impaired people. In addition, there have been studies on various types of walking assistive devices for visually impaired people. Sharmila et al. ${ }^{(7)}$ developed a goggles-type walking assistive device that can support the walking of visually impaired people through object detection and identification using a camera, earphones, principal component analysis (PCA) algorithm, GPS module, and microcontroller. However, it required complex calculations and was only effective in bright lighting. Nivash and Ganesh ${ }^{(8)}$ studied a glove-type walking assistive device that uses gloves, Arduino, ultrasonic sensors, IR sensors, and a Bluetooth module. However, the method of using a glove rather than a cane is unfamiliar to visually impaired people. There have been many other studies on walking assistive devices for visually impaired people, but most of them had problems with the cost and recognizing the user's location. ${ }^{(9-11)}$ Accordingly, it is necessary to develop a cost-effective device with various functions such as obstacle detection, location recognition, and the provision of safety in dark environments by considering usability regardless of age and country. ${ }^{(12-14)}$

In this study, we developed a cost-effective walking assistive cane that can recognize the current location for visually impaired people. To provide environmental information, which is translated into haptic signals to the user when obstacles are detected to the front and the left and right sides when walking, ultrasonic sensors and a vibration motor were used in this device. A radio frequency identification (RFID) system, a speaker, a recording module, and an automatic lighting system (comprising a cadmium sulfide (CdS) photoresistor and an LED) were also utilized to recognize the current location and to identify a user in dark environments. Considering the increased weight of the cane due to the use of various sensors, a wheel was attached to the end of the cane to allow the user to use the cane in a push-forward manner.

\section{Method}

\subsection{Device design}

As shown in Fig. 1, we developed a walking assistive cane based on a traditional white cane. The model names and manufacturers of the modules used are described in Table 1. As shown in 

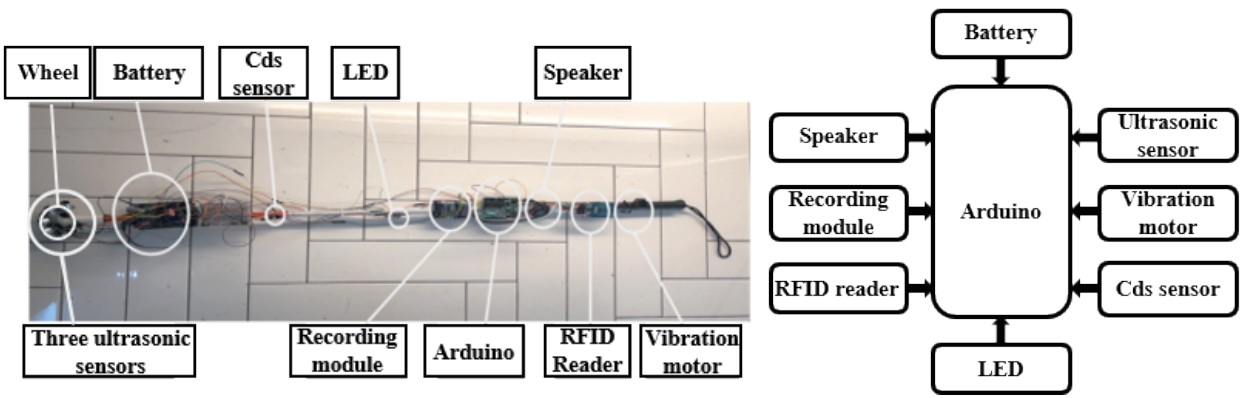

(a)

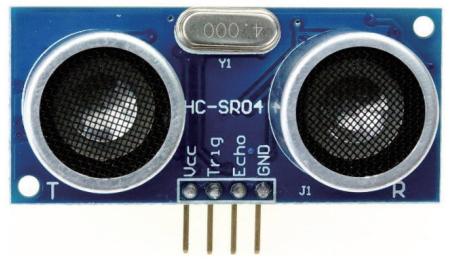

(b)

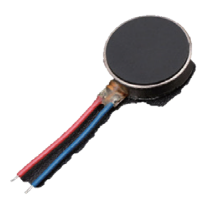

(c)



(d)

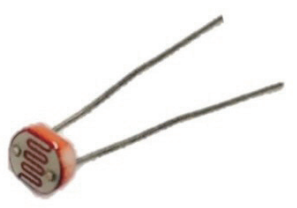

(g)

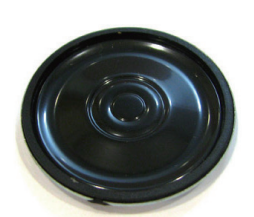

(e)

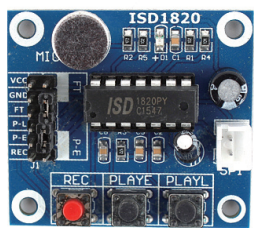

(f)



(h)

(i)

Fig. 1. (Color online) (a) Device design, (b) ultrasonic sensor, (c) vibration motor, (d) RFID system, (e) speaker, (f) recording module, (g) CdS photoresistor, (h) LED, and (i) Arduino.

Table 1

Module specification.

\begin{tabular}{lcc}
\hline Name & Model & Manufacturer \\
\hline Ultrasonic sensor & HC-SR04 & Elecfreaks (China) \\
Vibration motor & 30073R & SEEED (China) \\
RFID reader & SZH-EK040 & SZH (China) \\
RFID tag & Mifare-S50 keychain & SZH (China) \\
Speaker & FDS400500 & FUDING (China) \\
Recording module & SZH-SDBJ-001 & SMG (China) \\
CdS photoresistor & GL5528 & Ardest (China) \\
LED & 8C4UC02 & DAKWANG (China) \\
Arduino & Arduino Mega & ARDUINO.CC (Italy) \\
\hline
\end{tabular}


Fig. 2, considering the usability and weight of the developed cane, it was designed to be pushed forward using a wheel attached to the tip rather than swung from side to side. On the basis of previous research and survey data for visually impaired people, the detection range of the developed cane was set to $1.2 \mathrm{~m}$ in front and $0.5 \mathrm{~m}$ to the side.

\section{A. Ultrasonic sensors}

Three ultrasonic sensors were attached to the bottom of the cane, perpendicular to the ground, for obstacle detection to the front, left, and right of the user. This sensor module, which operated with a $5 \mathrm{~V}$ power source, included the ultrasonic transmitters, the receiver, and the control circuit. The developed cane was designed to sense low-lying obstacles on the ground using the ultrasonic sensors with a measuring angle of $15^{\circ}$.

\section{B. Vibration motor}

A vibration motor, which was attached to the handle of the cane, was used to deliver information regarding the obstacle distance in a haptic feedback manner to the user. To detect obstacles accurately in advance, the vibration motor was designed to vibrate at $9000 \mathrm{rpm}$ at $3 \mathrm{~V}$ when there are obstacles within $30 \mathrm{~cm}$.

\section{RFID system}

An RFID system was used to provide information about the user's location in indoor spaces. This system was composed of an RFID reader, two tags, a speaker, and a recording module. The RFID reader operated at $3.3 \mathrm{~V}$ and can detect a tag within a distance of about $5 \mathrm{~cm}$, allowing users to intuitively recognize their location. It was attached to the front of the handle of the cane for fast and effective tag identification. When the RFID reader detects the backscattered signal from a tag, a voice message announcing the user's current location is sent to the user using the speaker attached on the cane.

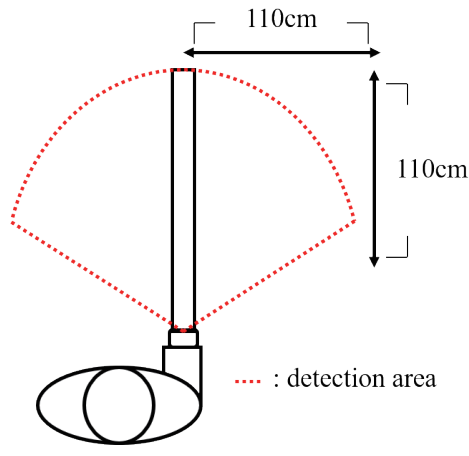

(a)

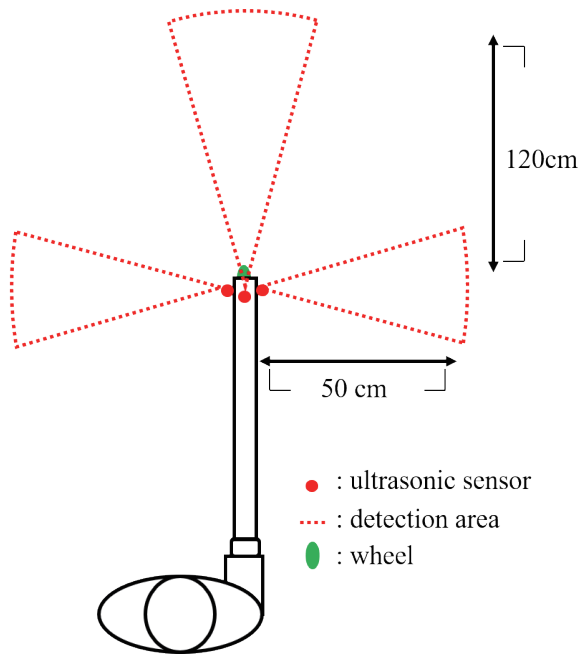

(b)

Fig. 2. (Color online) Differences in detection range between (a) white cane and (b) developed walking assistive cane. 


\section{Automatic lighting system}

An automatic lighting system composed of a CdS photoresistor and an LED was attached on the middle part of the cane for the safety of the user. The CdS photoresistor, which can measure ambient light in units of 10 lux, was utilized to assess the light intensity level and the LED was turned on when the sensor detected a low light intensity level, thus alerting both the user and nearby sighted people to the risk of collision.

\section{E. Arduino}

All controls were performed via an Arduino Mega module, which was selected as the cheapest module satisfying the performance required for this study. It was operated with a 7-12 $\mathrm{V}$ power source comprising six $1.5 \mathrm{~V}$ batteries for long-term use. The tip of the cane was changed to a wheel to reduce the burden of its weight on the user.

\subsection{Experiment}

In this study, ten participants with normal vision were recruited from Jeonbuk National University in Jeonju, Republic of Korea. None of the participants had gait abnormality or history of injury in the lower extremities of the musculoskeletal system. All participants gave written informed consent regarding the purpose and experimental protocol of this study. To evaluate the performance of the developed cane, the experiment was carried out in an area of $10 \mathrm{~m}$ length and $2.2 \mathrm{~m}$ width, as shown in Fig. 3. Small cylindrical obstacles of $11.5 \mathrm{~cm}$ diameter and $9.2 \mathrm{~cm}$ height were placed within the area. These obstacles represented low bollards, stone roots, and thresholds, which are the most common causes of falls for visually impaired people. These obstacles were made of non-woven fabric materials to prevent injury caused by collision while walking. RFID tags were attached to the walls of the area as starting and ending points, allowing users to recognize their current location.

The experiment was conducted under two conditions: (1) walking with a traditional white cane and (2) walking with the developed walking assistive cane. In particular, the starting position was randomly set to one of three regions (left, middle, or right side) to prevent the

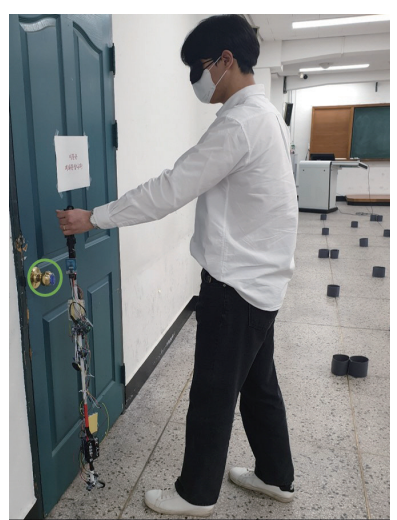

(a)

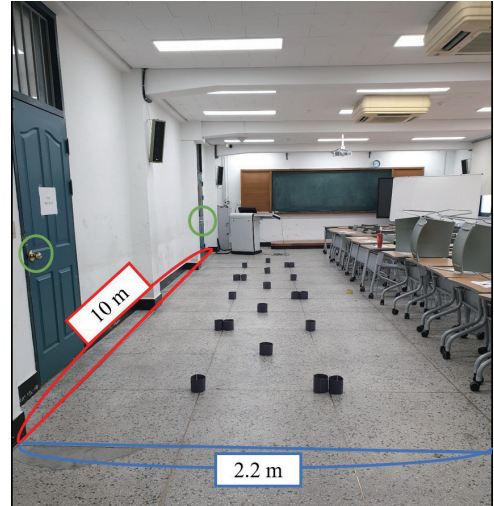

(b)

Fig. 3. (Color online) (a) Experimental setup and (b) RFID tag contact (green circles = RFID tags). 
recognition of the obstacle locations as a result of repeated experiments. Before the experiment, all participants walked on normal ground for 5 min to warm up and relax their bodies. Then, they were instructed to put on a blindfold and walk at a comfortable speed along the area with obstacles. An assistant also walked behind the participants for safety. The experiments were repeated three times and participants took a 5 min rest between experiments to prevent fatigue. The required time and the number of collisions were measured to investigate the effect of the developed cane on the gait. A survey including five questions on the satisfaction of walking with the developed cane (RFID tag response time, RFID tag reading distance, obstacle recognition, ease of identification of location, and ease of use) was conducted after the experiment.

\subsection{Data analysis}

SPSS 12.0 (IBM Corp., Chicago, USA) software was used to perform statistical analysis. To determine the normality of all variables, a Shapiro-Wilk normality test was conducted. The oneway analysis of variance (ANOVA) with Tukey's post hoc test was performed to compare the differences in the required time and the number of collisions. The Mann-Whitney $U$ test was also used to compare the variables between the two experimental conditions at the $p<0.05$ level.

\section{Results}

\subsection{Time required and number of collisions}

Table 2 and Fig. 4(a) show the times required under the two experimental conditions. When walking with the white cane, the average time required was $44.76 \mathrm{~s}$. The times required in the second and third tests were 9.23 and $12.79 \%$ lower than that required in the first test, respectively. The average time required among all tests was $60.59 \mathrm{~s}$ when walking with the developed walking assistive cane. The average times required in the second and third tests were 9.58 and $10.4 \%$ longer than that required in the first test when walking with the walking assistive cane, respectively. There were no significant differences in the time required among all three tests.

Table 2

Time required and number of collisions in each test.

\begin{tabular}{llccc}
\hline & & White cane & Developed walking assistive cane & $p$-value \\
\hline \multirow{3}{*}{ Time required (s) } & 1st & 48.31 & 56.81 & 0.121 \\
\cline { 2 - 5 } & 2nd & 43.85 & 62.25 & 0.113 \\
\cline { 2 - 5 } & 3rd & 42.13 & 62.72 & 0.112 \\
\cline { 2 - 5 } & Average & 44.76 & 60.59 & 0.118 \\
\hline \multirow{3}{*}{$\begin{array}{l}\text { Number of collision } \\
\text { (number) }\end{array}$} & $1 \mathrm{st}$ & 2.30 & 1.70 & 0.152 \\
\cline { 2 - 5 } & 2nd & 1.90 & 2.00 & 0.114 \\
\cline { 2 - 5 } & Average & 2.00 & 1.70 & 0.121 \\
\hline
\end{tabular}

$(M \pm S D) * p<0.05$ 


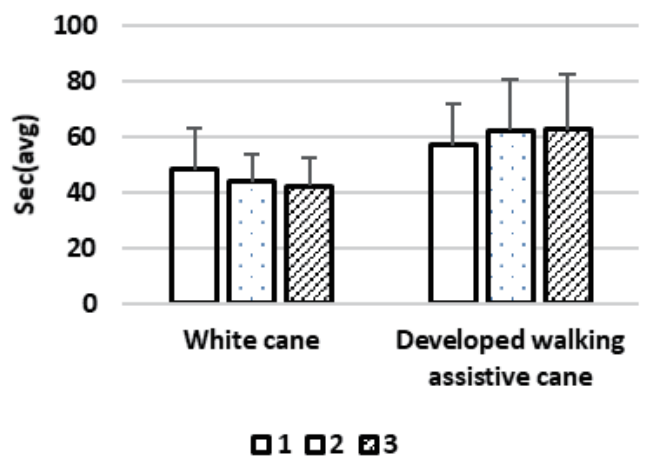

(a)

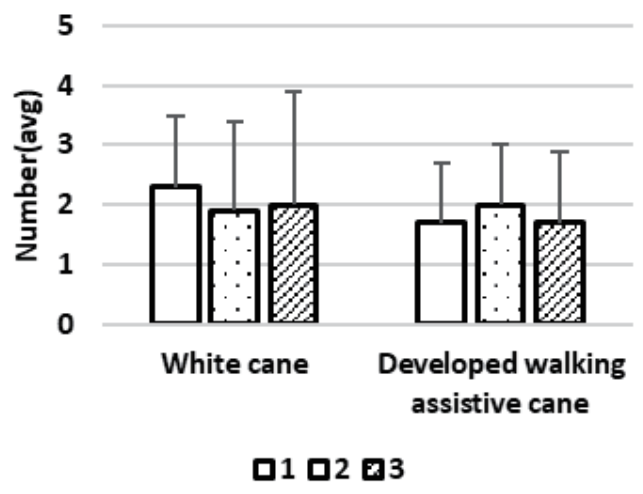

(b)

Fig. 4. (a) Time required and (b) number of collisions in each test.

Overall, the time required was longer when using the developed walking assistive cane than when using the white cane. However, the difference in the time required was not significant.

Table 2 and Fig. 4(b) show the numbers of collisions under the two experimental conditions. When walking with the white cane, the average number of collisions among all tests was 2.1, and the numbers of collisions in the second and third tests were 17.39 and $13.04 \%$ less than that in the first test when walking with the white cane, respectively. The average number of collisions among all tests was 1.8 when walking with the developed assistive cane, and the number of collisions in the second test was $17.65 \%$ more than that in the first test. There were no significant differences in the number of collisions among all three tests. Overall, the number of collisions was lower when using the developed walking assistive cane than when using the white cane but the difference was not significant.

\subsection{Satisfaction}

Figure 5 shows the user satisfaction with the functions of the developed walking assistive cane. Concerning the RFID tag response time, $70 \%$ of users were satisfied or very satisfied and $30 \%$ were neutral. Regarding the RFID tag perception distance with the developed walking assistive cane, $90 \%$ of users were satisfied or very satisfied and $10 \%$ were neutral. In contrast, only $30 \%$ of users were satisfied or very satisfied with the obstacle recognition of the developed walking assistive cane while $60 \%$ were neutral.

Figure 6 shows the user satisfaction with ease of use and ease of identification of the location. Concerning ease of identification of the location, $60 \%$ of users were satisfied or very satisfied and $40 \%$ were neutral when using the developed walking assistive cane. In contrast, when walking with the white cane, only $10 \%$ were satisfied, $50 \%$ were neutral, and $40 \%$ were dissatisfied with ease of identification of the location. Concerning ease of use, when walking with the developed cane, $60 \%$ of users were satisfied or very satisfied and $40 \%$ were neutral. On the other hand, only $10 \%$ were satisfied when walking with the white cane, $40 \%$ were neutral, and $50 \%$ were dissatisfied with ease of use. 


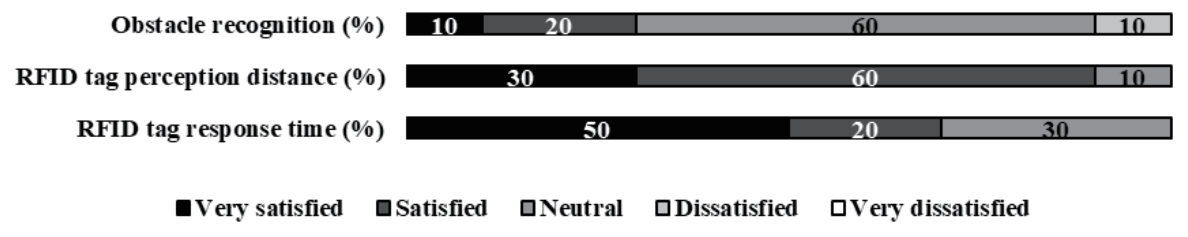

Fig. 5. User satisfaction with functions of the developed cane.

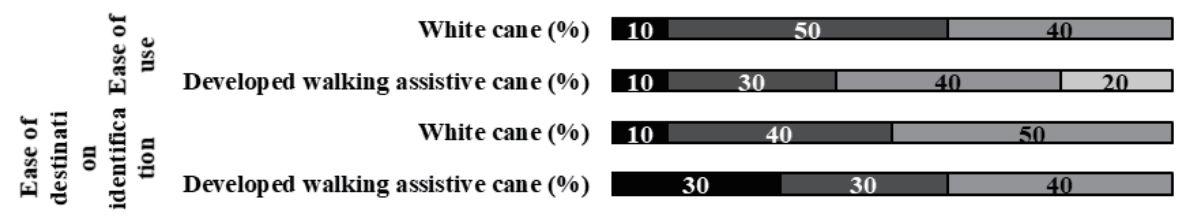

Fig. 6. User satisfaction with ease of use and identification of location.

\section{Discussion}

In this study, a walking assistive cane with an ultrasonic sensor, a vibration motor, an RFID system, a CdS photoresistor, an LED, and a speaker was developed for visually impaired people. To evaluate the effectiveness of the developed cane, the time required and number of collisions were compared for the cases of walking with the white cane and walking with the developed walking assistive cane in an area with small cylindrical obstacles. In addition, a survey was carried out to determine the level of user satisfaction with the developed cane.

It was found that a longer time was required when walking with the developed cane than when walking with the white cane. It is believed that this was due to a delay in information transmission when using the developed cane. Commonly, users can only obtain information on obstacles in front by swinging the white cane while walking. To overcome this limitation, the developed cane was designed to simultaneously detect information on objects to the front, left, and right using three ultrasound sensors and provide a vibrating alarm to the user when obstacles were detected on the ground. However, the user walked slowly due to the risk of collision after receiving the notification of the obstacles. In addition, it was difficult to accurately transmit information on the direction of obstacles with a single vibration motor. Contrary to the results for the time required, the number of collisions was reduced when walking with the developed cane. In a previous study, it was reported that the number of visually impaired people tends to increase gradually from middle age, and the prevalence of falls is higher among older adults with low vision. ${ }^{(15)}$ Indeed, falls are the major cause of low self-confidence and serious injuries in visually impaired people. ${ }^{(16)}$ In other words, it is very important to consider various methods of preventing falls for visually impaired people. Previous studies have reported that lighting is the easiest way to reduce the increased risk of pedestrians on dark roads. ${ }^{(17)}$ However, it is difficult for blind people to distinguish between bright and dark environments, then turn on lighting. Accordingly, in this study, an automatic lighting system was installed on the developed 
cane to help visually impaired people walk safely even in dark environments. A system that automatically emits light according to the brightness of the surrounding environment can help reduce the risk of accidents that may occur to visually impaired people. Thus, the result of this study that the number of collisions was reduced when using the developed cane is very meaningful in improving the quality of life of visually impaired people.

The RFID system attached to the developed cane was used to aid the orientation of the participants in the experiment. Before the experiment, all participants were told the locations of RFID tags, which were installed at the start and end points. In the survey, the location recognition function using the RFID system of the developed cane provided high satisfaction in both the perception distance and response time. In general, when a blind person walks with a white cane, they rely only on the soles of the feet, the sense of touch, and residual vision to orient themselves to their surroundings. Since most of these methods are based on experience in a familiar environment, there are limitations in accurately identifying a user's location in a new environment. When walking with the developed cane in this study, the current location can be more accurately identified than when walking with the white cane because information about the user's current location can be obtained through the stored voice when contacting the RFID tags. Accurate location identification can have a positive effect on improving life satisfaction as well as increasing the area of activity for visually impaired people. Accordingly, RFID technologies and their application methods that can be used in walking assistive devices for visually impaired people were proposed in previous studies. ${ }^{(18-20)}$ Despite the advantage of being able to provide accurate information about the start and end points, the characteristic of the RFID system that tags can only be recognized when the distance between the reader and tag is within $5 \mathrm{~cm}$ may be a limitation in its use. Another disadvantage is that it is difficult to acquire location information while walking. For safety, it is essential to increase the recognition distance in order to provide information on the user's current location and direction even while walking. Several authors have proposed other technologies with a relatively long recognition distance compared with that of the RFID system. ${ }^{(21,22)}$ Therefore, it is necessary to evaluate the effect of location recognition using a beacon on the gait of visually impaired people in future studies.

\section{Conclusions}

It is very important to develop a walking assistive device for visually impaired people to prevent falls and provide a safe walking environment by detecting obstacles on the ground in advance and accurately identifying the current location. In this study, we confirmed that a costeffective walking assistive cane, which consists of three ultrasonic sensors, a vibration motor, an RFID system, a CdS photoresistor, an LED, and a speaker, could help realize safe walking and accurate position recognition in a new environment. The results of this study can be applied to improving the satisfaction of spatial perception ability when transmitting spatial information to visually impaired people using RFID, and can be used to develop electronic travel aids in the future. In a future study, we will attempt to develop a method that can deliver information on obstacles to the user more intuitively and quickly while walking than the current walking assistive device. Additionally, a study on wireless communication technology and applications 
that can provide useful information for visually impaired people such as current location, walking direction, walking speed, and distance to the destination will be conducted.

\section{Acknowledgments}

This work was supported by a National Research Foundation of Korea (NRF) grant funded by the Korea government (MSIT) (No. NRF-2019R1A2C1008454).

\section{References}

1 B. Lee and Y. Lee: Disabil Health J. 12 (2019) 79. https://doi.org/10.1016/j.dhjo.2018.07.012

2 T. Campisi, M. Ignaccolo, G. Inturri, G. Tesoriere and V. Torrisi: Res. Transp. Bus. Manag. 40 (2021) 100592. https://doi.org/10.1016/j.rtbm.2020.100592

3 Y. Lin, K. Wang, W. Yi, S. Lian: Proc. IEEE/CVF Int. Conf. Computer Vision Workshops (IEEE, 2019).

4 S. Shoval, I. Ulich, and J. Borenstein: IEEE Robot. Autom. Mag. 10 (2003) 9. https://doi.org/10.1109/ MRA.2003.1191706

5 M. Wahab, A. Talib, H. Kadir, A. Johari, A. Noraziah, R. Sidek, and A. Mutalib: Int. J. Comput. Sci. Issues 8 (2011) 21. https://arxiv.org/pdf/1110.5156

6 B. Hoyle and D. Waters: Assistive Technology for Visually Impaired and Blind People (Springer, London, 2008) pp. 209-229

7 V. Sharmila, N. Paul, P. Ezhumalai, S. Reetha, and S. Kumar: Mater. Today Proc. 2 (2020). https://doi. org/10.1016/j.matpr.2020.10.198

8 S. Nivash and E. Ganesh: Mater. Today Proc. 9 (2021) https://doi.org/10.1016/j.matpr.2020.10.847

9 R. Tapu, B. Mocanu, and T. Zaharia: Pattern Recognit. Lett. 137 (2020) 37. https://doi.org/10.1016/j. patrec.2018.10.031

10 C. S. Silva and P. Wimalarntne: Sens. Mater. 31 (2019) 1305. https://doi.org/10.18494/SAM.2019.2232

11 C. S. Silva and P. Wimalaratne: Sens. Mater. 32 (2020) 1497. https://doi.org/10.18494/SAM.2020.2646

12 V. Graham and K. Dovorany: J. Bodyw. Mov. Ther. 20 (2016) 104. https://doi.org/10.1016/j.jbmt.2015.06.012

13 D. Nandini and K. R. Seeja: J. King Saud Univ. Comput. Inf. Sci. 31 (2019) 385. https://doi.org/10.1016/j. jksuci.2017.03.005

14 S. Maidenbaum, S. Abboud, and A. Amedi: Neurosci. Biobehav. Rev. 41 (2014) 3. http://dx.doi.org/10.1016/j. neubiorev.2013.11.007

15 S. Park, I. Choi, S. Kim, and S. Kim: Infrared. Phys. Technol. 67 (2014) 583. https://doi.org/10.1016/j. infrared.2014.09.023

16 U. Sait, V. Ravishankar, T. Kumar, R. Bhaumik, G. Lal, K. Bhalla, and K. sanjay: Procedia. Comput. Sci. 167 (2020) 2244. https://doi.org/10.1016/j.procs.2020.03.277

17 J. Griswold, B. Fishbain, S. Washington, and D. Ragland: Accid. Anal. Prev. 43 (2011) 301. https://doi. org/10.1016/j.aap.2010.08.028

18 K. Yelamarthi, D. Haas, D. Nielsen, and S. Motherse: 2010 53rd IEEE Int. Midwest Symp. Circuits and Systems (IEEE, 2010) pp. 1149-1152.

19 V. Kulyukin, C. Gharpure, and J. Nicholson: 2005 IEEE/RSJ Int. Conf. Intelligent Robots and Systems (IEEE, 2005) pp. 2845-2850.

20 D. Lopez-de-Ipina, T. Lorido, and U. Lopez: Ambient Assisted Living IWAAL 2011 (Springer, Berlin, 2011).

21 D. Ahmetovic, C. Gleason, C. Ruan, K. Kitani, H. Takagi, and C. Asakawa: Proc. 18th Int. Conf. HumanComputer Interaction with Mobile Devices and Services (Association for Computing Machinery, 2016) 90-99.

22 T. Ishihara, J. Vongkulbhisal, K. Kitani, and C. Asakawa: 2017 IEEE Winter Conf. Applications of Computer Vision (WACV) (IEEE, 2017) 769-777 


\section{About the Authors}

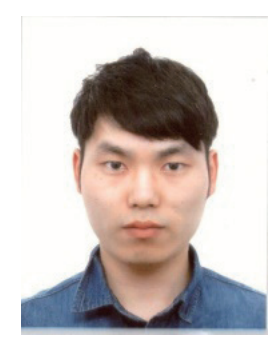

Chang-Min Yang received his B.S. degree from Chonbuk National University, South Korea, in 2016. His major research interests are wearable devices for visually impaired people, gait analysis, and functional shoes. (whycm@jbnu.ac.kr)

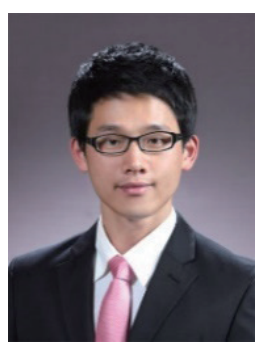

Ji-Yong Jung received his B.S., M.S., and Ph.D. degrees from Chonbuk National University, South Korea, in 2010, 2012, and 2016, respectively. He is currently a research assistant professor at Chonbuk National University. His major research interests are postural balance, gait analysis, and rehabilitation. (cholbun@hanmail.net)

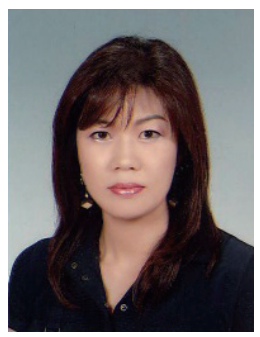

Jung-Ja Kim received her B.S. and M.S. degrees in 1985 and 1988, respectively, and her Ph.D. degree in 2002, all in computer science, from Chonnam National University, South Korea. She worked at the Electronic Telecommunication Laboratory at Chonnam National University from 2002 to 2004 and at the Korea Bio-IT Foundry Center at Gwangju from 2004 to 2006. Since 2006, she has been a professor at Chonbuk National University. Her major research interests are big data analysis (biological and medical data), artificial intelligence, and rehabilitation. (jungjakim@jbnu.ac.kr) 
